\title{
Clinical Interventions to Improve Nutritional Care in Older Adults and Patients in Primary Healthcare - A Scoping Review of Current Practices of Health Care Practitioners
}

\author{
Sonja Lindner-Rabl (D) \\ Valentina Wagner (D) \\ Andreas Matijevic' \\ Carolin Herzog ' \\ Christina Lampl ${ }^{\prime}$ \\ Julia Traub ${ }^{2}$ \\ Regina Roller-Wirnsberger (D) \\ 'Medical University of Graz, Department \\ of Internal Medicine, Research Unit for \\ Aging and Old Age Medicine, Graz, \\ Austria; ${ }^{2}$ University Hospital Graz, \\ Department of Clinical Medical \\ Nutrition, Graz, Austria
}

\begin{abstract}
In light of the increasing life expectancy of Europe's population and the rising significance of active and healthy ageing relating thereto, an integrated approach of nutritional care within primary health care is gaining importance. The aim of the review was to summarize evidence on the effectiveness of nutritional interventions in primary health care. The scoping review is based upon a comprehensive literature search of relevant literature published between January 2010 and August 2021 in PubMed, CINAHL, Cochrane Database of Systematic Reviews, Embase and Medline databases. Overall, 15 studies were included for evidence synthesis and interventions were basically clustered according to their type, into 1) eHealth and tele-medical interventions; 2) targeted single interventions; and 3) comprehensive, multi-faceted interventions. The review presents diverging evidence regarding the efficacy and effectiveness of interventions for nutritional care in primary health care, however, demonstrates encouraging outcomes. eHealth and tele-medical interventions partly show a careful positive tendency. Likewise, manifold single interventions on patient level present significant improvements in patient health outcomes. Multifaceted and comprehensive interventions found in the literature also partly demonstrate significant changes in intervention groups. Primary health care represents a critical setting for the care of older citizens and patients with complex health needs. This scoping review provides an overview of current nutrition care practices in primary health care and results reinforce the need to strengthen implementation of multi-faceted interventions carried out by the inter-disciplinary primary care team for advanced nutritional care.
\end{abstract}

Keywords: malnutrition, older people, nutritional care, primary care

\section{Introduction}

In the last century, Europe's population structure has changed significantly resulting in an ageing population, living longer than ever with a current life expectancy of 77.7 years for men and 84.3 years for women. ${ }^{1}$ This increase in life expectancy is one of the most remarkable societal achievements in the history of mankind, however, a considerable amount of life is being spent with disabilities and diseases impacting the quality of life. These circumstances underline the need to put the concept of healthy ageing increasingly into perspective. ${ }^{1}$ Healthy ageing as a comprehensive approach does not raise the claim of being free of disease or impairment, as up to $95 \%$ of the primary care population aged 65 and over are
Correspondence: Regina Roller-Wirnsberger Medical University of Graz, Department of Internal Medicine, Research Unit for Aging and Old Age Medicine, Auenbruggerplatz I5, Graz, 8036, Austria

Tel +43316385 12274

Fax +4331638513062

Email Aging-ukim@medunigraz.at 
affected by multimorbidity. ${ }^{2}$ However, the World Health Organization (WHO) defines healthy ageing as a "process of developing and maintaining the functional ability that enables well-being in older age". ${ }^{3}$ The decade of healthy ageing 2021-2030 has been launched by the WHO recently and the need for person-centered integrated care with primary care as a central element represents one "action area" to approach healthy ageing. ${ }^{4,5}$ To meet these holistic needs of older adults, health systems need to be transformed, which results in the requirement of fundamental changes in the way care is designed and delivered. ${ }^{4}$ The primary health care approach plays a key role in the implementation of these requirements. ${ }^{5}$

The ageing process is associated with physiological and psychological changes that affect the capacity of older people, and their health issues become more chronic and complex. ${ }^{5,6}$ Among other nutritional challenges, an increased risk of malnutrition ${ }^{5,7}$ which is also associated with poor quality of life, reduced functional ability and premature mortality ${ }^{8}$ occur in older adults. Additionally, there are reasonable grounds to support the hypothesis that social and economic factors are associated with malnutrition and malnutrition risk in older adults. ${ }^{9}$ The prevalence of high malnutrition risk in Europe depends on the screening tool used and varies among countries and across health care settings with a prevalence of $28.0 \%$ in hospitals, $17.5 \%$ in residential care, and $8.5 \%$ in community settings. However, pooled prevalence rates of high malnutrition amount to $23 \%$ of European older adults. ${ }^{10}$

In its consensus statement, the European Society of Clinical Nutrition and Metabolism (ESPEN) presented two sets of criteria for diagnosing malnutrition, after patients at risk are correctly identified by a validated screening tool. The first criteria is a Body-Mass-Index (BMI) $<18.5 \mathrm{~kg} / \mathrm{m}^{2}$. The second option is unintentional weight loss of $>10 \%$ indefinite of time or $>5 \%$ over the last 3 months in combination with either BMI $<20 \mathrm{~kg} / \mathrm{m}^{2}$ for patients aged over 70 years, or $<22 \mathrm{~kg} / \mathrm{m}^{2}$ for patients aged up to 70 years, or a fat free mass index (FFMI) of $<$ $15 \mathrm{~kg} / \mathrm{m}^{2}$ in women and $17 \mathrm{~kg} / \mathrm{m}^{2}$ in men. Moreover, malnutrition should be considered a condition that could occur in all aberrant nutritional states. ${ }^{11}$

Only few data exist that show the evidence for structured nutritional interventions and nutrition care pathways in primary care, although the primary health care setting plays a key role in delivering targeted nutritional interventions, as primary health care providers, as major contact point, are regarded well-placed to provide nutritional care. ${ }^{12}$ In view of fragmented and diverging nutrition policies within the European Union (EU), striving for a common and integrated approach of nutritional care within active and healthy ageing seems reasonable. ${ }^{13}$ It was therefore the aim of the current paper to pull together evidence on this topic and prepare a manuscript for healthcare professionals working in primary care. Further uptake of nutritional care, especially for older patients, will be facilitated as insights on current interventions are given, ranging from particular interventions on micro-level, up to eHealth interventions and comprehensive, versatile interventions.

\section{Materials and Methods}

The review presented in this publication was conducted according to PRISMA guidelines for SCOPE reviews. ${ }^{14}$

\section{Data Resources and Search Strategy}

Relevant studies published between January 2010 and August 2021 in English language were identified using PubMed, CINAHL, Cochrane Database of Systematic Reviews, Medline and Embase databases. The applied search strategy comprised the following Medical Subject Headings: "aged" AND ("nutrition therapy" OR "nutrition policy" OR "nutrition assessment" OR "diet, food and nutrition" OR "Elder Nutritional Physiological Phenomena") AND "Primary Health Care". If necessary, the medical subject headings were adapted to specific database options by using synonyms of the Medical Subject Headings. Further search via reference tracking was performed to identify additional studies.

\section{Inclusion Criteria}

To be included, publications had to meet all of the following criteria aligned with PICO criteria for developing a research question: ${ }^{15} 1$ ) population: older, geriatric patients $\geq 60$ years in the primary care setting or upon hospital discharge with nutritional status assessed; 2) intervention: nutritional interventions and/or nutritional care relating to malnutrition prevention or management; 3) comparison: usual care/no intervention; 4) outcome: relevant nutritional, dietetic, clinical or behavioral outcomes. Study designs considered for inclusion were randomized controlled trials or non-randomized controlled trials, cross-sectional studies, cohort studies or case-control studies published between 2010 and 2021.

\section{Outcomes of Interest}

The outcome of interest was the impact of interventions relating to nutritional care or nutritional status, functional 
status or other patient health outcomes in regards to malnutrition such as hospital (re-)admissions and mortality.

\section{Data Synthesis and Analysis According to Nutritional Care for Malnourished \\ Patients}

Full-text screening was performed by two persons and disagreements about inclusion of literature were discussed among researchers until consensus was reached. Relevant outcome data from the included studies were descriptively summarized and clustered according to their intervention nature. Based on the expected heterogeneity and complexity of the results, this review is presented as scoping review.

\section{Results}

A total of 1309 relevant citations were identified through search strategy. 7 additional studies were detected by hands-on search. After removing duplicates $(n=365$ articles removed), title-/abstract screening ( $n=875$ articles removed) and full-text screening ( $\mathrm{n}=61$ articles removed), 15 studies met the inclusion criteria. The PRISMA diagram $^{16}$ illustrates the selection process of the studies and shows reasons for exclusion (Figure 1).

\section{Study Characteristics}

The study designs were divided into eleven randomized controlled trials, ${ }^{17-27}$ one partially randomized controlled trial$^{28}$ one randomized comparative study, ${ }^{29}$ one controlled feasibility trial, ${ }^{30}$ and one cross-sectional study. ${ }^{31}$

All studies included had been conducted in Europe, covering the following countries in particular: Denmark $(\mathrm{n}=5),{ }^{17,20,22,24,30}$ The Netherlands $(\mathrm{n}=3),{ }^{18,23,27}$ Spain $(\mathrm{n}=2),{ }^{19,21}$ Lithuania $(\mathrm{n}=1),{ }^{31}$ Finland $(\mathrm{n}=1),{ }^{29}$ Germany $(n=1),{ }^{25}$ Sweden $(n=1),{ }^{26}$ and Israel $(n=1) .{ }^{28}$

Analyzed study settings ranged from community and/ or primary care $(n=6),{ }^{21,23,26,28,29,31}$ upon hospital discharge $(n=8),{ }^{17,18,20,22,24,25,27,30}$ or home care setting $(\mathrm{n}=1){ }^{19}$

The study population considered (total $n=1778$ ) comprised older adults at risk of malnutrition or undernutrition, ${ }^{17,20,22-25,27-30}$ older adults defined as frail, ${ }^{26}$ older caregiver-dependent adults, ${ }^{19}$ and older adults without further characterization. ${ }^{18,21,31} 14$ studies out of 15 indicated a higher ratio of females in the study population, ranging from a share of $55.2 \%{ }^{27}$ up to $83.1 \%{ }^{20}$ of females. Table 1 illustrates the descriptive study characteristics.

\section{Interventions Addressing Malnutrition and Impact on Older People's Health Outcomes}

Figure 2 shows an overview of intervention types found during this literature search. The characteristics of the interventions applied are manifold, ranging from the implementation of eHealth and tele-medical systems; ${ }^{25,30}$ the utilization of targeted single interventions on microlevel; ${ }^{17,18,20,23,24,27,28,31}$ and the provision of comprehensive, multi-faceted interventions. ${ }^{19,21,22,26,29}$

\section{eHealth and Tele-Medical Interventions}

A study analyzing the effect of a tele-medical monitoring system with daily assessments of patient health indicators showed no significant changes in weight, BMI or weight loss, ${ }^{25}$ whereas an intervention combining enriched meals and a technology-based self-monitoring application showed a positive tendency toward the intervention group in muscle strength, depression score, and healthrelated quality of life. ${ }^{30}$

\section{Targeted Single Interventions on Micro-Level}

Particular interventions that were found in the literature comprise individual nutritional counseling/care plans with different intensity, ${ }^{20,24,28,31}$ partly in combination with the prescription of oral nutritional supplements (ONS) and/or the provision of protein-enriched meals. ${ }^{17,18,23,27}$

Nutritional follow-up care by a dietician via home visit significantly decreased the risk of hospital readmission 30 days after discharge $(\mathrm{p}=0.03)$ and 90 days after discharge $(\mathrm{p}<0.01)$ compared to the control group, ${ }^{20}$ whereas in another study, nutritional follow-up care pointed toward a positive tendency of reduced risk of readmission in the control group. ${ }^{24}$ The intervention showed a significant effect in weight change $(\mathrm{p}=0.035)$, energy intake $(\mathrm{p}<0.001)$, protein intake $(\mathrm{p}=0.001)$ and ONS intake $(\mathrm{p}<0.001){ }^{24}$

A nutritional intervention led by dieticians as case managers showed a significant improvement in the followup nutritional status in the intervention group $(\mathrm{p}<0.05)$, with lower costs of primary care physicians' visits $(p=0.005)$ and specialists visits $(p=0.03)$ and significant improvement in depression score and cognitive function score $(\mathrm{p}=0.04){ }^{28}$ Spirgienè et $\mathrm{al}^{31}$ found similar positive follow-up effects when considering the impact of nutritional recommendations. After following nutritional 

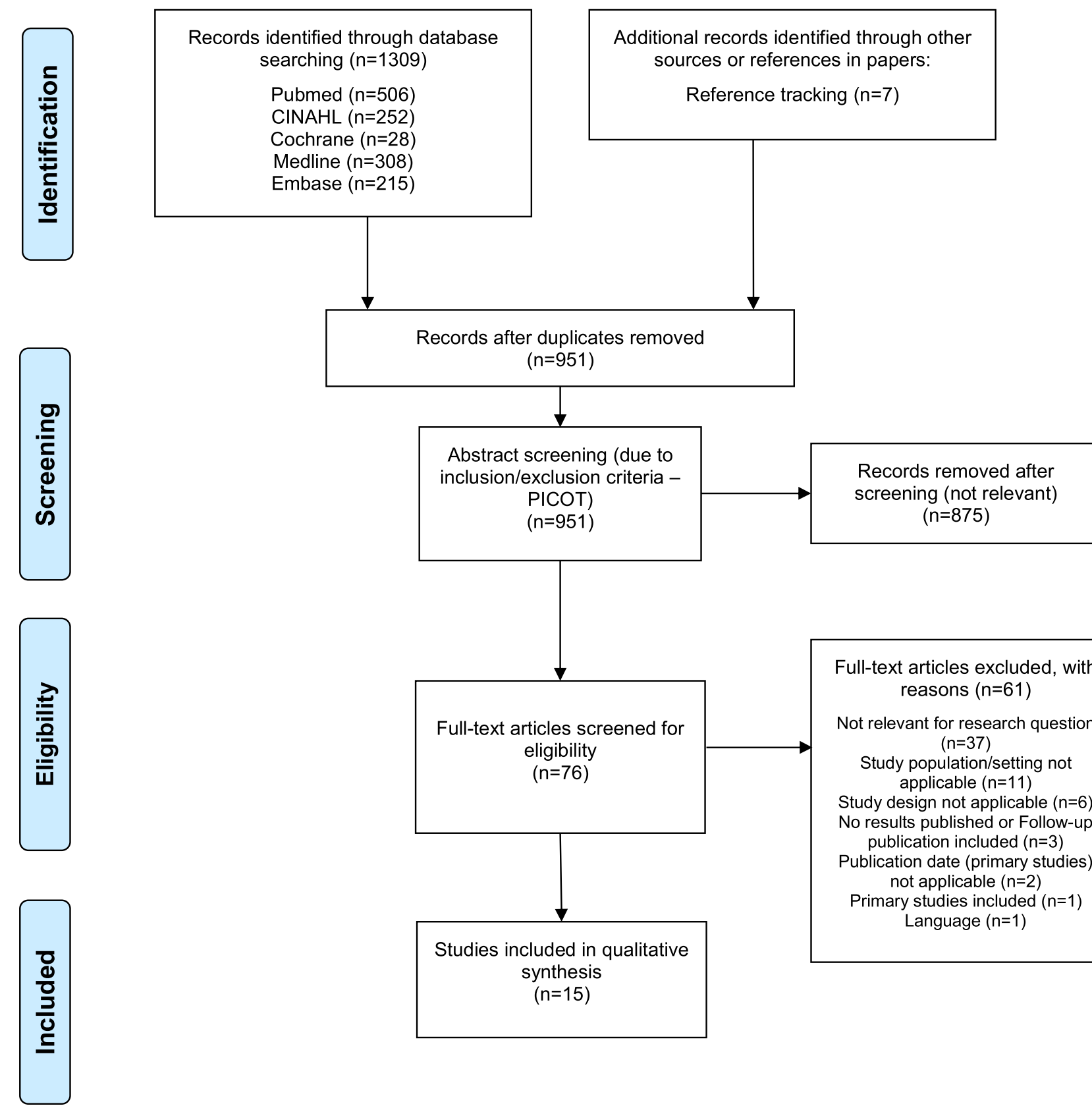

Full-text articles excluded, with reasons $(n=61)$

Not relevant for research question $(n=37)$

Study population/setting not applicable $(n=11)$

Study design not applicable $(n=6)$

No results published or Follow-up publication included $(n=3)$

Publication date (primary studies) not applicable $(n=2)$

Primary studies included $(n=1)$ Language $(n=1)$

Figure I PRISMA flow diagram.

Notes: This figure illustrates the search strategy applied. A total of 1316 records ( 1309 records by database searching and 7 records by reference tracking) were identified. Following the abstract and full-text screening process, 15 studies finally met the inclusion criteria and were further processed. PRISMA figure adapted from Moher D, Liberati A, Altman D, Tetzlaff J, et al. The PRISMA statement for reporting systematic reviews and meta-analyses of studies that evaluate health care interventions: explanation and elaboration. Journal of clinical epidemiology. 2009;62(10). Creative Commons. ${ }^{16}$

recommendations, the MNA scores of the study sample increased significantly $(\mathrm{p}<0.001){ }^{31}$

Developing individual nutritional care plans and providing nutritional care led to a significant change in body weight between groups $(\mathrm{p}<0.05)$ and the intervention group showed a significant increase in self-rated health $(p<0.05) .{ }^{17}$ The provision of protein-enriched foods and drinks led to a significant increase in mean protein intake in the intervention group $(\mathrm{p}<0.01)$ and to improved functional outcomes, body weight, and nutritional status in both groups compared to baseline parameters $(\mathrm{p}<0.05) .{ }^{18}$

In contrast to that, dietetic consultation and treatment with ONS, if needed, showed no significant follow-up effect on any outcomes in body weight, physical performance, handgrip strength, energy and protein intake, whole-body resistance or fat-free mass. ${ }^{23}$ Another 
Table I Study Characteristics

\begin{tabular}{|c|c|c|c|c|c|c|c|c|c|}
\hline $\begin{array}{l}\text { Author } \\
\text { (Year) }\end{array}$ & Country & $\begin{array}{l}\text { Study } \\
\text { Design }\end{array}$ & Setting & Duration & $\begin{array}{l}\text { Study } \\
\text { Population and } \\
\text { Assessment } \\
\text { Tool }\end{array}$ & $\begin{array}{c}\text { Sex } \\
\text { Ratio }\end{array}$ & Type of Intervention & $\begin{array}{l}\text { Primary } \\
\text { Outcome of } \\
\text { Interest }\end{array}$ & Primary Results \\
\hline \multirow{2}{*}{$\begin{array}{l}\text { Spirgiene } \\
\text { et al } \\
(2018)^{31}\end{array}$} & \multirow[t]{2}{*}{ Lithuania } & \multirow{2}{*}{$\begin{array}{l}\text { Cross- } \\
\text { Sectional } \\
\text { Descriptive } \\
\text { Study }\end{array}$} & \multirow[t]{2}{*}{$\begin{array}{l}\text { Primary } \\
\text { health care }\end{array}$} & \multirow[t]{2}{*}{6 months } & $\begin{array}{l}\text { Older people } \geq 65 \\
\text { years }(n=169)\end{array}$ & \multirow[t]{2}{*}{$\begin{array}{l}63.9 \% \\
\text { female }\end{array}$} & \multirow{2}{*}{$\begin{array}{l}\text { Single intervention on micro level: } \\
\text { changes in nutritional status after } \\
\text { following nutritional recommendations }\end{array}$} & \multirow[t]{2}{*}{ MNA score } & \multirow[t]{2}{*}{$\begin{array}{l}\text { Significant increase }(p<0.001) \text { in } \\
\text { whole study sample }\end{array}$} \\
\hline & & & & & $\begin{array}{l}\text { Mini Nutritional } \\
\text { Assessment } \\
\text { (MNA) }\end{array}$ & & & & \\
\hline \multirow[t]{2}{*}{$\begin{array}{l}\text { Terp et al } \\
(2018)^{17}\end{array}$} & \multirow[t]{2}{*}{ Denmark } & \multirow[t]{2}{*}{$\begin{array}{l}\text { Randomized } \\
\text { Controlled } \\
\text { Trial }\end{array}$} & \multirow[t]{2}{*}{$\begin{array}{l}\text { Hospital } \\
\text { discharge }\end{array}$} & \multirow[t]{2}{*}{3 months } & $\begin{array}{l}\text { Geriatric patients } \\
\geq 65 \text { years at } \\
\text { nutritional risk } \\
(n=144)\end{array}$ & \multirow[t]{2}{*}{$\begin{array}{l}77.8 \% \\
\text { female }\end{array}$} & \multirow[t]{2}{*}{$\begin{array}{l}\text { Single intervention on micro level: } \\
\text { individual dietary plan and three follow- } \\
\text { up visits vs usual care }\end{array}$} & \multirow[t]{2}{*}{$\begin{array}{l}\text { Change in body } \\
\text { weight }\end{array}$} & \multirow[t]{2}{*}{$\begin{array}{l}\text { Significant weight change between } \\
\text { groups }(p<0.05) \text { from baseline to } \\
\text { follow-up }\end{array}$} \\
\hline & & & & & $\begin{array}{l}\text { Nutritional Risk } \\
\text { Screening (NRS- } \\
\text { 2002) }\end{array}$ & & & & \\
\hline \multirow[t]{2}{*}{$\begin{array}{l}\text { Beelen et al } \\
(2017)^{18}\end{array}$} & \multirow[t]{2}{*}{$\begin{array}{l}\text { The } \\
\text { Netherlands }\end{array}$} & \multirow{2}{*}{$\begin{array}{l}\text { Randomized } \\
\text { Controlled } \\
\text { Trial }\end{array}$} & \multirow[t]{2}{*}{$\begin{array}{l}\text { Hospital } \\
\text { discharge }\end{array}$} & \multirow[t]{2}{*}{3 months } & $\begin{array}{l}\text { Older adults } \geq 65 \\
\text { years }(n=75)\end{array}$ & \multirow[t]{2}{*}{$\begin{array}{l}56 \% \\
\text { female }\end{array}$} & \multirow{2}{*}{$\begin{array}{l}\text { Single intervention on micro level: } \\
\text { protein-enriched foods and drinks vs } \\
\text { regular variants }\end{array}$} & \multirow{2}{*}{$\begin{array}{l}\text { Protein Intake; } \\
\text { Physical } \\
\text { performance } \\
\text { (SPPB) }\end{array}$} & \multirow[t]{2}{*}{$\begin{array}{l}\text { Increased mean protein intake in } \\
\text { intervention group }(p<0.01)\end{array}$} \\
\hline & & & & & $\begin{array}{l}\text { Mini Nutritional } \\
\text { Assessment } \\
\text { (MNA), 24-hour } \\
\text { recall and dietary } \\
\text { food record }\end{array}$ & & & & \\
\hline \multirow{3}{*}{$\begin{array}{l}\text { Fernández- } \\
\text { Barrés et al } \\
(2017)^{19}\end{array}$} & \multirow[t]{3}{*}{ Spain } & \multirow{3}{*}{$\begin{array}{l}\text { Randomized } \\
\text { Controlled } \\
\text { Trial }\end{array}$} & \multirow[t]{3}{*}{ Home Care } & \multirow[t]{3}{*}{$\begin{array}{l}12 \\
\text { months }\end{array}$} & $\begin{array}{l}\text { Older caregiver- } \\
\text { dependent adults }\end{array}$ & \multirow[t]{3}{*}{$\begin{array}{l}68.2 \% \\
\text { female }\end{array}$} & \multirow{3}{*}{$\begin{array}{l}\text { Comprehensive, multi-faceted } \\
\text { intervention: } \\
\text { educational session of caregivers and } \\
\text { patients and individual dietary } \\
\text { monitoring of the patient vs regular } \\
\text { home care visits }\end{array}$} & \multirow[t]{3}{*}{ MNA score } & \multirow[t]{3}{*}{$\begin{array}{l}\text { Significant increase of MNA score in } \\
\text { intervention group }(p<0.001)\end{array}$} \\
\hline & & & & & $\begin{array}{l}\geq 65 \text { years at } \\
\text { nutritional risk } \\
(n=173)\end{array}$ & & & & \\
\hline & & & & & $\begin{array}{l}\text { Mini Nutritional } \\
\text { Assessment } \\
\text { (MNA) }\end{array}$ & & & & \\
\hline
\end{tabular}


Table I (Continued).

\begin{tabular}{|c|c|c|c|c|c|c|c|c|c|}
\hline $\begin{array}{l}\text { Author } \\
\text { (Year) }\end{array}$ & Country & $\begin{array}{l}\text { Study } \\
\text { Design }\end{array}$ & Setting & Duration & $\begin{array}{c}\text { Study } \\
\text { Population and } \\
\text { Assessment } \\
\text { Tool }\end{array}$ & $\begin{array}{c}\text { Sex } \\
\text { Ratio }\end{array}$ & Type of Intervention & $\begin{array}{l}\text { Primary } \\
\text { Outcome of } \\
\text { Interest }\end{array}$ & Primary Results \\
\hline \multirow[t]{2}{*}{$\begin{array}{l}\text { Lindegaard } \\
\text { Pedersen } \\
\text { et al } \\
(2017)^{20}\end{array}$} & \multirow[t]{2}{*}{ Denmark } & \multirow[t]{2}{*}{$\begin{array}{l}\text { Randomized } \\
\text { Controlled } \\
\text { Trial }\end{array}$} & \multirow[t]{2}{*}{$\begin{array}{l}\text { Hospital } \\
\text { discharge }\end{array}$} & \multirow[t]{2}{*}{3 months } & $\begin{array}{l}\text { Malnourished } \\
\text { geriatric patients } \\
\text { and patients at risk } \\
\text { of malnutrition } \geq \\
75 \text { years }(n=208)\end{array}$ & \multirow[t]{2}{*}{$\begin{array}{l}83.1 \% \\
\text { female }\end{array}$} & \multirow[t]{2}{*}{$\begin{array}{l}\text { Single intervention on micro level: } \\
\text { nutritional follow-up care by a clinical } \\
\text { dietician via home visit or telephone call } \\
\text { vs no follow-up care }\end{array}$} & \multirow[t]{2}{*}{$\begin{array}{l}\text { Hospital } \\
\text { readmission at } 30 \\
\text { and } 90 \text { days }\end{array}$} & \multirow[t]{2}{*}{$\begin{array}{l}\text { Lower risk of readmission in home- } \\
\text { visit group compared to control } \\
\text { group } 30 \text { days after discharge } \\
(p=0.03) \text { and } 90 \text { days after discharge } \\
(p<0.01)\end{array}$} \\
\hline & & & & & $\begin{array}{l}\text { Mini Nutritional } \\
\text { Assessment } \\
\text { (MNA) }\end{array}$ & & & & \\
\hline \multirow[t]{2}{*}{$\begin{array}{l}\text { Lindhardt } \\
\text { et al } \\
(2017)^{30}\end{array}$} & \multirow[t]{2}{*}{ Denmark } & \multirow[t]{2}{*}{$\begin{array}{l}\text { Mixed- } \\
\text { Method } \\
\text { Study } \\
\text { (Controlled } \\
\text { Feasibility } \\
\text { Trial) }\end{array}$} & \multirow[t]{2}{*}{$\begin{array}{l}\text { Hospital } \\
\text { discharge }\end{array}$} & \multirow[t]{2}{*}{3 months } & $\begin{array}{l}\text { Older patients } \geq \\
65 \text { years with } \\
\text { a risk for } \\
\text { malnutrition } \\
(n=36)\end{array}$ & \multirow[t]{2}{*}{$\begin{array}{l}\text { Not } \\
\text { specified }\end{array}$} & \multirow[t]{2}{*}{$\begin{array}{l}\text { eHealth/Telemonitoring intervention: } \\
\text { delivery of enriched meals and self- } \\
\text { monitoring vs usual care }\end{array}$} & \multirow[t]{2}{*}{$\begin{array}{l}\text { Muscle strength; } \\
\text { BMI }\end{array}$} & \multirow[t]{2}{*}{$\begin{array}{l}\text { Increase of muscle strength in } \\
\text { intervention group }\end{array}$} \\
\hline & & & & & $\begin{array}{l}\text { Nutritional Risk } \\
\text { Screening (NRS- } \\
\text { 2002) }\end{array}$ & & & & \\
\hline \multirow[t]{2}{*}{$\begin{array}{l}\text { Badia et al } \\
(2015)^{21}\end{array}$} & \multirow[t]{2}{*}{ Spain } & \multirow[t]{2}{*}{$\begin{array}{l}\text { Randomized } \\
\text { Controlled } \\
\text { Trial }\end{array}$} & \multirow[t]{2}{*}{$\begin{array}{l}\text { Primary } \\
\text { health care }\end{array}$} & \multirow[t]{2}{*}{2 years } & $\begin{array}{l}\text { Community- } \\
\text { dwelling } \\
\text { inhabitants born in } \\
1924(n=328)\end{array}$ & \multirow[t]{2}{*}{$\begin{array}{l}61.6 \% \\
\text { female }\end{array}$} & \multirow{2}{*}{$\begin{array}{l}\text { Comprehensive, multi-faceted } \\
\text { intervention: } \\
\text { community-based multifactorial } \\
\text { program via a treatment plan based on } \\
\text { a specific algorithm vs usual health care }\end{array}$} & \multirow[t]{2}{*}{ MNA score } & \multirow[t]{2}{*}{$\begin{array}{l}\text { No significant effects in primary } \\
\text { outcomes }\end{array}$} \\
\hline & & & & & $\begin{array}{l}\text { Mini Nutritional } \\
\text { Assessment } \\
\text { (MNA) }\end{array}$ & & & & \\
\hline \multirow[t]{2}{*}{$\begin{array}{l}\text { Beck et al } \\
(2015)^{22}\end{array}$} & \multirow[t]{2}{*}{ Denmark } & \multirow[t]{2}{*}{$\begin{array}{l}\text { Randomized } \\
\text { Controlled } \\
\text { Trial }\end{array}$} & \multirow[t]{2}{*}{$\begin{array}{l}\text { Hospital } \\
\text { discharge }\end{array}$} & \multirow[t]{2}{*}{3 months } & $\begin{array}{l}\text { Geriatric patients } \\
\geq 70 \text { years and at } \\
\text { nutritional risk } \\
(\mathrm{n}=71)\end{array}$ & \multirow[t]{2}{*}{$\begin{array}{l}67.6 \% \\
\text { female }\end{array}$} & \multirow{2}{*}{$\begin{array}{l}\text { Comprehensive, multi-faceted } \\
\text { intervention: } \\
\text { Discharge Liaison-Team in cooperation } \\
\text { with a dietician with three home visits } \\
\text { and an individual nutritional care plan vs } \\
\text { discharge liaison-team }\end{array}$} & \multirow[t]{2}{*}{ Nutritional status } & \multirow[t]{2}{*}{$\begin{array}{l}\text { Significant changes in weight } \\
(p=0.025) \text {, energy intake }(p=0.02) \\
\text { and protein intake }(p=0.003)\end{array}$} \\
\hline & & & & & $\begin{array}{l}\text { Nutritional Risk } \\
\text { Screening (NRS- } \\
\text { 2002) }\end{array}$ & & & & \\
\hline
\end{tabular}




\begin{tabular}{|c|c|c|c|c|c|c|c|c|c|}
\hline $\begin{array}{l}\text { Nykänen } \\
\text { et al } \\
(2014)^{29}\end{array}$ & Finland & $\begin{array}{l}\text { Randomized } \\
\text { Comparative } \\
\text { Study }\end{array}$ & Community & 2 years & $\begin{array}{l}\begin{array}{l}\text { Older people at } \\
\text { risk of malnutrition } \\
\geq 75 \text { years }(n=173)\end{array} \\
\text { Mini Nutritional } \\
\text { Assessment } \\
\text { (MNA) }\end{array}$ & $\begin{array}{l}68.8 \% \\
\text { female }\end{array}$ & $\begin{array}{l}\text { Comprehensive, multi-faceted } \\
\text { intervention: } \\
\text { individually tailored comprehensive } \\
\text { geriatric intervention, consisting of } \\
\text { a nutritional intervention, a medical and } \\
\text { physical intervention vs normal medical } \\
\text { care }\end{array}$ & MNA score & $\begin{array}{l}\text { Significant difference in MNA score } \\
\text { between groups after adjustment } \\
\text { for age, gender, IADL, MMSE and } \\
\text { FCI }\end{array}$ \\
\hline \multirow[t]{2}{*}{$\begin{array}{l}\text { Schilp et al } \\
(2013)^{23}\end{array}$} & \multirow[t]{2}{*}{$\begin{array}{l}\text { The } \\
\text { Netherlands }\end{array}$} & \multirow[t]{2}{*}{$\begin{array}{l}\text { Randomized } \\
\text { Controlled } \\
\text { Trial }\end{array}$} & \multirow[t]{2}{*}{$\begin{array}{l}\text { Primary } \\
\text { health care }\end{array}$} & \multirow[t]{2}{*}{6 months } & $\begin{array}{l}\text { Older people } \geq 65 \\
\text { years identified as } \\
\text { undernourished } \\
(n=146)\end{array}$ & \multirow[t]{2}{*}{$\begin{array}{l}64.4 \% \\
\text { female }\end{array}$} & \multirow[t]{2}{*}{$\begin{array}{l}\text { Single intervention on micro level: } \\
\text { dietetic treatment by a qualified trained } \\
\text { dietician vs usual care }\end{array}$} & \multirow[t]{2}{*}{$\begin{array}{l}\text { Body weight; } \\
\text { Physical } \\
\text { performance; } \\
\text { Hand-grip strength; }\end{array}$} & \multirow[t]{2}{*}{$\begin{array}{l}\text { No treatment effect on primary and } \\
\text { secondary outcomes }\end{array}$} \\
\hline & & & & & $\begin{array}{l}\text { Short Nutritional } \\
\text { Assessment } \\
\text { Questionnaire 65+ } \\
\text { (SNAQ) }\end{array}$ & & & & \\
\hline \multirow[t]{2}{*}{$\begin{array}{l}\text { Beck et al } \\
(2013)^{24}\end{array}$} & \multirow[t]{2}{*}{ Denmark } & \multirow[t]{2}{*}{$\begin{array}{l}\text { Randomized } \\
\text { Controlled } \\
\text { Trial }\end{array}$} & \multirow[t]{2}{*}{$\begin{array}{l}\text { Hospital } \\
\text { discharge }\end{array}$} & \multirow[t]{2}{*}{$\begin{array}{l}6.5 \\
\text { months }\end{array}$} & $\begin{array}{l}\text { Geriatric medical } \\
\text { patients } \geq 65 \text { years } \\
\text { at nutritional risk } \\
(n=152)\end{array}$ & \multirow[t]{2}{*}{\begin{tabular}{|l|}
$73 \%$ \\
female
\end{tabular}} & \multirow{2}{*}{$\begin{array}{l}\text { Single intervention on micro level: } \\
\text { individualized nutritional counseling by } \\
\text { a registered dietician complemented } \\
\text { with three follow-up visits by GPs } \\
\text { (general practitioners) vs three follow- } \\
\text { up visits by GPs alone }\end{array}$} & \multirow[t]{2}{*}{ Risk of readmission } & \multirow[t]{2}{*}{$\begin{array}{l}\text { Risk of readmission showed } \\
\text { a positive tendency toward control } \\
\text { group }(P=0.07)\end{array}$} \\
\hline & & & & & $\begin{array}{l}\text { Nutritional Risk } \\
\text { Screening (NRS- } \\
\text { 2002) }\end{array}$ & & & & \\
\hline $\begin{array}{l}\text { Kraft et al } \\
(2012)^{25}\end{array}$ & Germany & $\begin{array}{l}\text { Randomized } \\
\text { Controlled } \\
\text { Trial }\end{array}$ & $\begin{array}{l}\text { Hospital } \\
\text { discharge }\end{array}$ & 6 months & $\begin{array}{l}\text { Malnourished } \\
\text { patients with } \\
\text { a need for caloric } \\
\text { ONS (mean age } \\
79.8 \text { years) ( } \mathrm{n}=26 \text { ) } \\
\begin{array}{l}\text { Nutritional Risk } \\
\text { Screening (NRS- } \\
2002 \text { ) }\end{array}\end{array}$ & $\begin{array}{l}61.5 \% \\
\text { female }\end{array}$ & $\begin{array}{l}\text { eHealth/Telemonitoring intervention: } \\
\text { telemedical monitoring vs usual care }\end{array}$ & $\begin{array}{l}\text { Follow-up weight; } \\
\text { Follow-up BMI; } \\
\text { weight loss }\end{array}$ & No significant effect \\
\hline
\end{tabular}

(Continued) 
Table I (Continued).

\begin{tabular}{|c|c|c|c|c|c|c|c|c|c|}
\hline $\begin{array}{l}\text { Author } \\
\text { (Year) }\end{array}$ & Country & $\begin{array}{l}\text { Study } \\
\text { Design }\end{array}$ & Setting & Duration & $\begin{array}{l}\text { Study } \\
\text { Population and } \\
\text { Assessment } \\
\text { Tool }\end{array}$ & $\begin{array}{c}\text { Sex } \\
\text { Ratio }\end{array}$ & Type of Intervention & $\begin{array}{l}\text { Primary } \\
\text { Outcome of } \\
\text { Interest }\end{array}$ & Primary Results \\
\hline \multirow[t]{2}{*}{$\begin{array}{l}\text { Lammes } \\
\text { et al } \\
(2012)^{26}\end{array}$} & \multirow[t]{2}{*}{ Sweden } & \multirow[t]{2}{*}{$\begin{array}{l}\text { Randomized } \\
\text { Controlled } \\
\text { Trial }\end{array}$} & \multirow[t]{2}{*}{ Community } & \multirow[t]{2}{*}{9 months } & $\begin{array}{l}\text { Older adults } \geq 75 \\
\text { years defined as } \\
\text { frail }(n=93)\end{array}$ & \multirow[t]{2}{*}{$\begin{array}{l}60 \% \\
\text { female }\end{array}$} & \multirow{2}{*}{$\begin{array}{l}\text { Comprehensive, multi-faceted } \\
\text { intervention: } \\
\text { individual nutritional advice and group } \\
\text { sessions on nutrition for older adults vs } \\
\text { physical training vs combined } \\
\text { intervention vs control group }\end{array}$} & \multirow{2}{*}{$\begin{array}{l}\text { Energy intake; } \\
\text { Resting Metabolic } \\
\text { Rate (RMR); Body } \\
\text { Composition }\end{array}$} & \multirow{2}{*}{$\begin{array}{l}\text { Significant increase of RMR in } \\
\text { training group after } 3 \text { months } \\
(p<0.05) \text {; Significant increase of } \\
\text { waist circumference in control } \\
\text { group after } 3 \text { months }(p<0.05)\end{array}$} \\
\hline & & & & & $\begin{array}{l}\text { Mini Nutritional } \\
\text { Assessment } \\
\text { (MNA), four-day } \\
\text { food record }\end{array}$ & & & & \\
\hline \multirow[t]{2}{*}{$\begin{array}{l}\text { Endevelt } \\
\text { et al } \\
(2011)^{28}\end{array}$} & \multirow[t]{2}{*}{ Israel } & \multirow[t]{2}{*}{$\begin{array}{l}\text { Partially } \\
\text { Randomized } \\
\text { Controlled } \\
\text { Trial }\end{array}$} & \multirow[t]{2}{*}{ Community } & \multirow[t]{2}{*}{6 months } & $\begin{array}{l}\text { Community- } \\
\text { dwelling patients } \geq \\
75 \text { years at } \\
\text { nutritional risk } \\
(\mathrm{n}=127)\end{array}$ & \multirow[t]{2}{*}{$\begin{array}{l}60-64 \% \\
\text { female }^{a}\end{array}$} & \multirow[t]{2}{*}{$\begin{array}{l}\text { Single intervention on micro level: } \\
\text { dietary intensive treatment vs medical } \\
\text { treatment vs standard care }\end{array}$} & \multirow[t]{2}{*}{$\begin{array}{l}\text { Nutritional status: } \\
\text { MNA score, } \\
\text { biochemical } \\
\text { measurements, } \\
\text { food frequency } \\
\text { questionnaire }\end{array}$} & \multirow[t]{2}{*}{$\begin{array}{l}\text { Significant improvement in dietetic } \\
\text { intervention treatment group in } \\
\text { dietary intake }\end{array}$} \\
\hline & & & & & $\begin{array}{l}\text { Mini Nutritional } \\
\text { Assessment -short } \\
\text { form (MNA-sf) }\end{array}$ & & & & \\
\hline \multirow[t]{2}{*}{$\begin{array}{l}\text { Neelemaat } \\
\text { et al } \\
(2011)^{27}\end{array}$} & \multirow[t]{2}{*}{$\begin{array}{l}\text { The } \\
\text { Netherlands }\end{array}$} & \multirow[t]{2}{*}{$\begin{array}{l}\text { Randomized } \\
\text { Controlled } \\
\text { Trial }\end{array}$} & \multirow[t]{2}{*}{$\begin{array}{l}\text { Hospital } \\
\text { discharge }\end{array}$} & \multirow[t]{2}{*}{3 months } & $\begin{array}{l}\text { Older } \\
\text { malnourished } \\
\text { patients } \geq 60 \text { years } \\
(n=210)\end{array}$ & \multirow[t]{2}{*}{$\begin{array}{l}55.2 \% \\
\text { female }\end{array}$} & \multirow{2}{*}{$\begin{array}{l}\text { Single intervention on micro level: } \\
\text { energy and protein enriched diet, two } \\
\text { additional servings of ONS, vitamin D3 } \\
\text { and calcium supplement and telephone } \\
\text { counseling by a dietician vs usual care }\end{array}$} & \multirow[t]{2}{*}{$\begin{array}{l}\text { Changes in ADL } \\
\text { (functional } \\
\text { limitations and } \\
\text { physical activity) }\end{array}$} & \multirow[t]{2}{*}{$\begin{array}{l}\text { Significant decrease in functional } \\
\text { limitations between groups in the } \\
\text { per protocol analysis }\end{array}$} \\
\hline & & & & & $\begin{array}{l}\text { BMI and } \\
\text { unintentional } \\
\text { weight loss }\end{array}$ & & & & \\
\hline
\end{tabular}

Note: a'Depending on group allocation. 


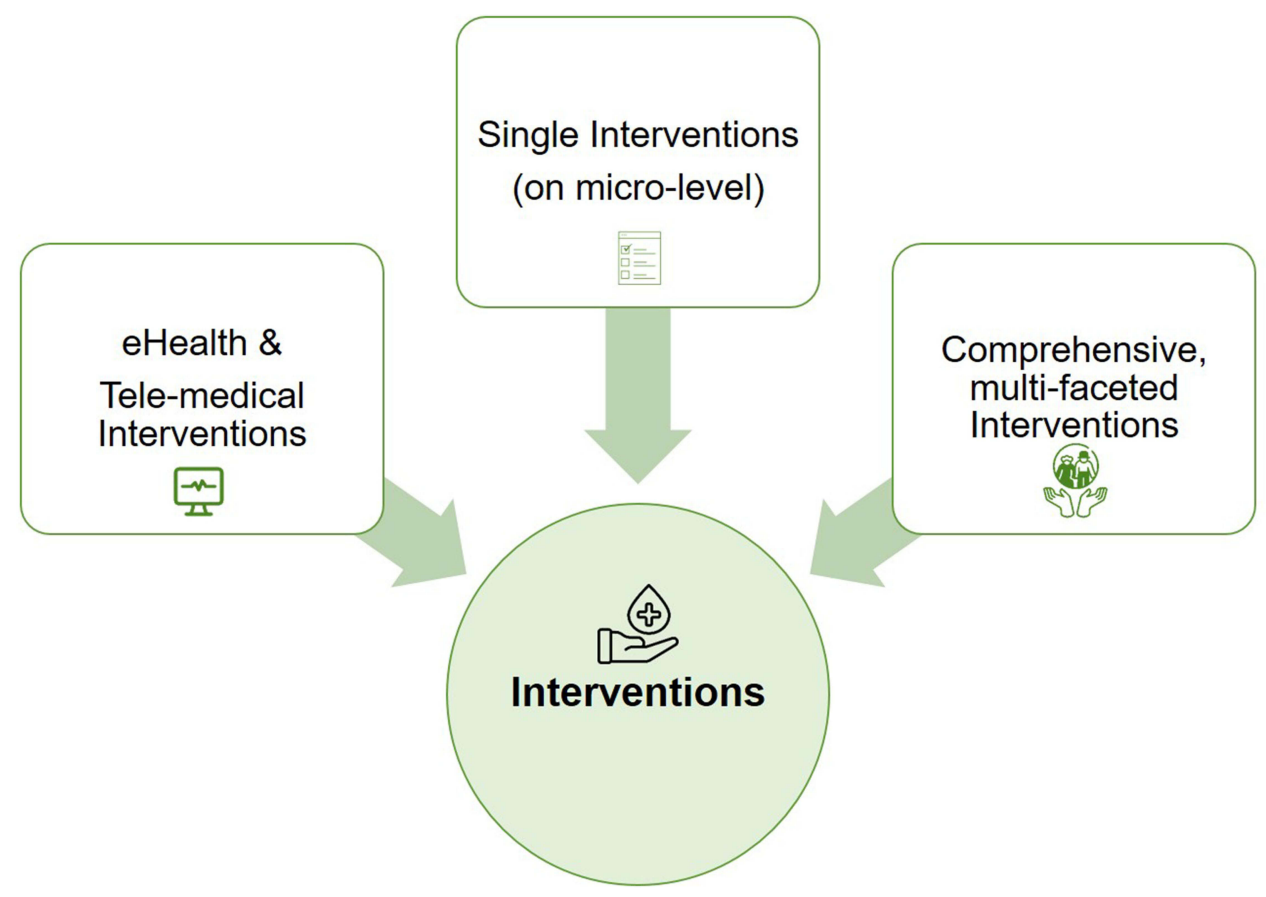

Figure 2 Overview of intervention types addressing malnutrition in community-dwelling older citizens.

Note: Summary of intervention types addressing malnutrition and/or risk of malnutrition in older community dwelling citizens.

intervention comprising an enriched diet, micronutrients supplementation, additional ONS servings and telephone counseling led to a significant increase in body weight in the highest body weight category in the intervention group (mean difference $+3.4 \mathrm{~kg}, 95 \%$ CI $0.2-6.6$ ). In the perprotocol analysis, a significant decrease in functional limitations in the intervention group was found. ${ }^{27}$

\section{Comprehensive, Multi-Faceted Interventions}

The implementation of comprehensive and multi-faceted interventions found in the literature included individual nutritional counseling in combination with physical activity ${ }^{26}$ a community-based multifactorial program based on a treatment plan with a specific algorithm; ${ }^{21}$ the inclusion of dieticians into a multi-professional discharge team; ${ }^{22}$ an individual comprehensive geriatric intervention, comprising of a nutritional, medical and physical intervention; ${ }^{29}$ and education and training of caregivers in combination with dietary monitoring of the patients. ${ }^{19}$

In a multi-faceted intervention study presenting four different treatment arms with individual dietary counseling and/or physical training, no significant changes in energy intake could be determined. However, after a period of 3 months, the training group showed a significant increase in resting metabolic rate $(p<0.05)$ and the control group showed a significant increase in waist circumference $(\mathrm{p}<0.05) .{ }^{26}$ Regarding the intervention using treatment algorithms, no significant changes in nutritional status determined by Mini Nutritional Assessment (MNA) only were found, ${ }^{21}$ whereas the inclusion of a dietician into a multi-professional discharge team had a positive effect on weight $(\mathrm{p}=0.025)$, protein intake $(\mathrm{p}=0.003)$ and a lower (re-) hospitalization rate after six months $(\mathrm{p}=0.017)$ in the intervention group. ${ }^{22}$

An individual comprehensive geriatric intervention consisting of a nutritional, medical and physical intervention also resulted in a significant difference of MNA score between groups after adjustment for age, gender, IADL (Instrumental Activities of Daily Living), MMSE (MiniMental State Examination) and FCI (Functional Comorbidity Index $)(p \leq 0.05){ }^{29}$

Fernández-Barrés et $\mathrm{al}^{19}$ demonstrated that a combined intervention of caregiver education and dietary monitoring of the patients resulted in an increased MNA score $(p<0.001)$, an increased consumption of eggs $(p=0.018)$, polyunsaturated fatty acids $(\mathrm{p}=0.006)$, folate $(\mathrm{p}=0.041)$ and vitamin $\mathrm{E}(\mathrm{p}=0.002)$ as well as improved nutritional knowledge $(p<0.001)$ in the intervention group. 


\section{Discussion}

The review presents diverging evidence regarding the efficacy and effectiveness of interventions to tackle the risk of malnutrition in older citizens and patients in primary healthcare. In some studies, a significant change or improvement in nutritional status could be demonstrated regardless of the nature of the intervention. ${ }^{17,19,22,24,28,29}$ Several publications showed contrasting results with no significant changes or improvement in nutritional status, ${ }^{21,23,25,26}$ however, other significant results could be determined in the field of healthcare use ${ }^{28}$ and hospital readmission, ${ }^{20}$ or self-rated health. ${ }^{17}$ Regarding nutritionrelated outcomes, the results of the studies showed a significant improvement through nutritional interventions including a dietician, ${ }^{22,24,28,31}$ protein enriched foods and drinks, ${ }^{18,27}$ comprehensive geriatric interventions, ${ }^{22}$ a multi-professional discharge team or a combined intervention of caregiver education and dietary monitoring. ${ }^{19}$

Primary care represents a critical setting for provision of nutritional care as an integral part of prevention and management of chronic conditions. ${ }^{32}$ Therefore, preventing and managing malnutrition in the community setting entails challenges to be considered within the health services. Perceived barriers toward addressing malnutrition properly by community health professionals are the lack of training for primary care practitioners, the probability of misconceptions about malnutrition being passed on to patients, a lack of time resources to weigh patients regularly in primary care and the difficulty to adapt an established eating behavior in older people ${ }^{33}$ as well as lack of systematically screening for malnutrition and a lack of support to deal with malnutrition in the community. ${ }^{34,35}$ Moreover, awareness about malnutrition and patterns of clinical interventions may differ across Europe, as it is also reflected in the composition of study countries within this review, with Denmark and The Netherlands offering rich insights in clinical interventions for nutritional care. Guidance to support well-nutrition care in older people across Europe is exemplarily provided by the European Aging Network. The guide "promoting well-nutrition in elderly care" presents key principles, processes and good practice examples to harmonize nutritional care and tackle malnutrition successfully. ${ }^{36}$ Effective responses for the management of malnutrition and the identification of patients at risk of malnutrition require support and strengthening of the primary care team, in which dieticians and community nurses serve as key stakeholders. ${ }^{34}$ The establishment of an integrated and multi-disciplinary primary care team facilitates collaborative and coordinated nutritional care and benefits the patient. ${ }^{35}$ The approach of professional integration is also considered in Valentijn's Rainbow Model of integrated care ${ }^{37}$ and, besides the creation of inter-disciplinary teams, also encompasses the importance of inter-professional education. When implementing a nutritional care intervention in the community, education programs for healthcare professionals should be provided within a multi-component strategy that is of benefit across all levels: for the patient, the interdisciplinary care team, and the health system. ${ }^{38}$ Supportive policies, plans and regulatory frameworks are needed to meet these requirements in primary health care. ${ }^{5}$

A significant amount of publications $(n=8)$ included in this review analyzed community-dwelling patients after hospital discharge. ${ }^{17,18,20,22,24,25,27,30}$ The transfer after hospital discharge back to the patient's home remains a critical period as a "nutritional gap" often inhibits full recovery, resulting in adverse effects on functional ability and rehabilitation. ${ }^{39}$ It has been shown recently, that nutritional variables indeed predict chances of returning home and activities of daily living in post-acute geriatric care. ${ }^{40}$ Furthermore, a multi-disciplinary transitional program ensuring the continuity of care, that not only improved nutritional status but also associated frailty status and functional ability, showed promising results in community-dwelling older adults. ${ }^{41}$ This triple effect is not really surprising, as the interlinkage between malnutrition and frailty is being substantially recognized and despite their diverging etiology both concepts share common ideas and measures. $^{42}$

As a result, malnutrition and risk of malnutrition represent a multi-faceted problem in the older population that needs to be reflected in primary care and clinical practice. ${ }^{16}$ By addressing the challenges that come along with malnutrition in older citizens, through raising awareness of the issue, developing care pathways, providing education and training facilities for healthcare professionals and promoting timely care interventions, effective responses to the complex needs of this vulnerable population group are facilitated. ${ }^{43}$ Treating malnutrition is not only improving patients' quality of life and health but also benefits the health care system. Malnutrition can have economic consequences due to the fact that patients with malnutrition who are not treated adequately are more likely to need health care. ${ }^{44,45}$ Primary health care can play a vital role in saving costs, 
decreasing the utilization of health care resources, and tackling the upcoming issue of an ageing population, who is more likely to be malnourished, through early identification and treatment of malnourished patients. An easy way to identify it is through screening patients at primary health care centers and care homes. ${ }^{44}$

The major strength of this scoping review is the broad and comprehensive search approach. It was therefore possible to give a comprehensive overview of evidence of interventions in malnutrition care of older people in primary health care. However, the evidence collected in this scoping review is not yet sufficiently convincing to prefer one intervention over another, especially when considering that some of the studies included analyzed older people not at risk of malnutrition or already malnourished. The broad spectrum of interventions included during the review does not allow highly sensitive comparisons of actions and outcomes. Many of the studies included rather give insight into context of good-practice approaches published earlier. This limitation is aggravated by the fact that no qualitative studies have been included for analysis within this scoping review. Especially in the field of nutrition and dietetics, qualitative research studies offer the potential of increasing the value of quantitative evidence review, as they deal with questions of human behavior and help to inform the development of robust interventions. ${ }^{46}$

Reflecting on the outcomes of this review, implications for clinical practice can be given. Dieticians should play an active and leading role in multimodal nutritional interventions in primary care, as this review presents promising effects of dietician-led interventions. Moreover, nutritional care should be tailored toward nutritional needs and preferences of patients to enable highly individualized and targeted interventions. Effective nutritional care in primary care presupposes a correct identification of patients at risk of malnutrition or already malnourished. This is facilitated by the use of standardized and validated screening tools. However, future research in this regard should emphasize further improvement mechanisms of nutritional care interventions in primary care.

\section{Conclusion}

This scoping review presents a synopsis of current practices in malnutrition care of older citizens. In conclusion, interventions presented have not consistently shown a beneficial effect for nutritional care. However, targeted and/or multimodal interventions majorly guided by dieticians as well as the design and conduct of individual nutritional care plans revealed promising results in terms of health-related patient outcomes. In contrast to that, effects of eHealth-based interventions remain vague and leave room for further generating evidence.

The overall pressure on healthcare systems to reduce costs and "re-tailor" systems toward preventive innovative and integrated care models indicate the urgent need to set up future studies and collaborative frameworks in the field of nutrition of older citizens in primary healthcare and create future evidence for person-centered nutritional care interventions. Associated therewith, supporting strategies for education and training of primary care health professionals to provide effective nutritional care within integrated treatment models need to be taken into account.

\section{Research Ethics and Consent}

This review does not use or reveal any personal or patient data.

\section{Acknowledgments}

The authors would like to thank the Competence Center for Integrated Care (CCIV) which was the contracting entity for a research project dedicated toward the development of a malnutrition care pathway.

\section{Funding}

This research received no external funding.

\section{Disclosure}

The authors report no conflicts of interest in this work.

\section{References}

1. Science Advice for Policy by European Academies (SAPEA). Transforming the future of ageing. Available from: https://doi.org/10. 26356/ageing. Accessed August 17, 2021.

2. Violan C, Foguet-Boreu Q, Flores-Mateo G, et al. Prevalence, determinants and patterns of multimorbidity in primary care: a systematic review of observational studies. PLoS One. 2014;9(7):e102149. doi:10.1371/journal.pone. 0102149

3. World Health Organization. World report on ageing and health. Available from: https://apps.who.int/iris/handle/10665/186463. Accessed August 17, 2021.

4. World Health Organization. Decade of healthy ageing: baseline report. Available from: https://www.who.int/publications/i/item/ 9789240017900. Accessed August 17, 2021.

5. World Health Organization. Integrated care for older people. Realigning primary health care to respond to population ageing. Available from: https://apps.who.int/iris/bitstream/handle/10665/ 326295/WHO-HIS-SDS-2018.44-eng.pdf?sequence=1\&isAllowed=y. Accessed September 30, 2021.

6. Wells JL, Dumbrell AC. Nutrition and aging: assessment and treatment of compromised nutritional status in frail elderly patients. Clin Interv Aging. 2006;1(1):67-79. doi:10.2147/ciia.2006.1.1.67 
7. World Health Organization. Integrated care for older people: guidelines on community-level interventions to manage declines in intrinsic capacity. Available from: https://www.who.int/publications/i/item/ 9789241550109. Accessed September 30, 2021.

8. Integrated care for older people (ICOPE). Guidelines on community-level interventions to manage declines in intrinsic capacity. Evidence profile: malnutrition; 2017. Available from: https:// www.who.int/ageing/health-systems/icope/evidence-centre/ICOPEevidence-profile-malnutrition.pdf?ua=1\#: :text=Undernutrition $\% 20$ is \%20common\%20among\%20older,\%2Dincome\%20countries\%20(6). Accessed September 30, 2021.

9. Besora-Moreno M, Llauradó E, Tarro L, Solà R. Social and economic factors and malnutrition or the risk of malnutrition in the elderly: a systematic review and meta-analysis of observational studies. Nutrients. 2020;12(3):737. doi:10.3390/nu12030737

10. Leij-Halfwerk S, Verwijs MH, van Houdt S, et al. Prevalence of protein-energy malnutrition risk in European older adults in community, residential and hospital settings, according to 22 malnutrition screening tools validated for use in adults $\geq 65$ years: a systematic review and meta-analysis. Maturitas. 2019;126:80-89. doi:10.1016/j. maturitas.2019.05.006

11. Cederholm T, Bosaeus I, Barazzoni R, et al. Diagnostic criteria for malnutrition - an ESPEN consensus statement. Clin Nutr. 2015;34 (3):335-340. doi:10.1016/j.clnu.2015.03.001

12. Kraef C, Wood B, von Philipsborn P, et al. Primary health care and nutrition. Bull World Health Organ. 2020;98:886-893. doi:10.2471/ BLT.20.251413

13. Illario M, Maione A, Rusciano M, et al. NutriLive: an integrated nutritional approach as a sustainable tool to prevent malnutrition in older people and promote active and healthy ageing-The EIP-AHA Nutrition Action Group. Adv Public Health. 2016;2016:1-9. doi:10.1155/2016/5678782

14. Tricco A, Lillie E, Zarin W, et al. PRISMA extension for scoping reviews (PRISMA-ScR): checklist and explanation. Ann Intern Med. 2018;169(7):467-473. doi:10.7326/M18-0850

15. Sackett D, Richardson W, Rosenburg W, Haynes R. How to Practice and Teach Evidence Based Medicine. 2nd ed. Churchill Livingstone; 1997.

16. Moher D, Liberati A, Tetzlaff J, Altman DG, The PG. Preferred reporting items for systematic reviews and meta-analyses: the PRISMA statement. PLoS Med. 2009;6(7):e1000097. doi:10.1371/ journal.pmed.1000097

17. Terp R, Jacobsen KO, Kannegaard P, Larsen A-M, Madsen OR, Noiesen E. A nutritional intervention program improves the nutritional status of geriatric patients at nutritional risk - a randomized controlled trial. Clin Rehabil. 2018;32(7):930-941. doi:10.1177/ 0269215518765912

18. Beelen J, de Roos NM, de Groot LC. A 12-week intervention with protein-enriched foods and drinks improved protein intake but not physical performance of older patients during the first 6 months after hospital release: a randomised controlled trial. Br J Nutr. 2017;117 (11):1541-1549. doi:10.1017/S0007114517001477

19. Fernández-Barrés S, García-Barco M, Basora J, Martínez T, Pedret R, Arija V. The efficacy of a nutrition education intervention to prevent risk of malnutrition for dependent elderly patients receiving Home Care: a randomized controlled trial. Int $J$ Nurs Stud. 2017;70:131-141. doi:10.1016/j.ijnurstu.2017.02.020

20. Lindegaard Pedersen J, Pedersen PU, Damsgaard EM. Nutritional follow-up after discharge prevents readmission to hospital a randomized clinical trial. J Nutr Health Aging. 2017;21(1):75-82. doi:10.1007/s12603-016-0745-7

21. Badia T, Formiga F, Ferrer A, Sanz H, Hurtos L, Pujol R. Multifactorial assessment and targeted intervention in nutritional status among the older adults: a randomized controlled trial: the Octabaix study. BMC Geriatr. 2015;15(1):45. doi:10.1186/s12877015-0033-0
22. Beck A, Andersen UT, Leedo E, et al. Does adding a dietician to the liaison team after discharge of geriatric patients improve nutritional outcome: a randomised controlled trial. Clin Rehabil. 2015;29 (11):1117-1128. doi:10.1177/0269215514564700

23. Schilp J, Kruizenga HM, Wijnhoven HAH, van Binsbergen JJ, Visser M. Effects of a dietetic treatment in older, undernourished, community-dwelling individuals in primary care: a randomized controlled trial. Eur J Nutr. 2013;52(8):1939-1948. doi:10.1007/s00394013-0495-9

24. Beck AM, Kjaer S, Hansen BS, Storm RL, Thal-Jantzen K, Bitz C. Follow-up home visits with registered dietitians have a positive effect on the functional and nutritional status of geriatric medical patients after discharge: a randomized controlled trial. Clin Rehabil. 2013;27 (6):483-493. doi:10.1177/0269215512469384

25. Kraft M, van den Berg N, Kraft K, et al. Development of a telemedical monitoring concept for the care of malnourished geriatric home-dwelling patients: a pilot study. Maturitas. 2012;72 (2):126-131. doi:10.1016/j.maturitas.2012.02.011

26. Lammes E, Rydwik E, Akner G. Effects of nutritional intervention and physical training on energy intake, resting metabolic rate and body composition in frail. a randomised, controlled pilot study. J Nutr Health Aging. 2012;16(2):162-167. doi:10.1007/s12603-0110157-7

27. Neelemaat F, Bosmans JE, Thijs A, Seidell JC, van Bokhorst-de van der Schueren MA. Post-discharge nutritional support in malnourished elderly individuals improves functional limitations. J Am Med Dir Assoc. 2011;12(4):295-301. doi:10.1016/j.jamda.2010.12.005

28. Endevelt R, Lemberger J, Bregman J, et al. Intensive dietary intervention by a dietitian as a case manager among community dwelling older adults: the EDIT study. $J$ Nutr Health Aging. 2011;15 (8):624-630. doi:10.1007/s12603-011-0074-9

29. Nykänen I, Rissanen TH, Sulkava R, Hartikainen S. Effects of individual dietary counseling as part of a comprehensive geriatric assessment (CGA) on nutritional status: a population-based intervention study. J Nutr Health Aging. 2014;18(1):54-58. doi:10.1007/ s12603-013-0342-y

30. Lindhardt T, Nielsen MH. Older patients' use of technology for a post-discharge nutritional intervention - a mixed-methods feasibility study. Int J Med Inform. 2017;97:312-321. doi:10.1016/j. ijmedinf.2016.10.017

31. Spirgienè L, Damulevičienè G, Tomkevičiūtė J, Riklikienè O. Nutritional status of rural community-dwelling older people and changes after following nutritional recommendations. Int $J$ Nurs Pract. 2018;24(6):e12688. doi:10.1111/ijn.12688

32. Royal Australian College of General Practitioners. Guidelines for Preventive Activities in General Practice. 8th ed. RACCP; 2012.

33. Avgerinou C, Bhanu C, Walters K, et al. Supporting nutrition in frail older people: a qualitative study exploring views of primary care and community health professionals. Br J Gen Pract. 2020;70(691): e138-e145. doi:10.3399/bjgp20X707861

34. Dominguez Castro P, Reynolds CME, Kennelly S, et al. General practitioners' views on malnutrition management and oral nutritional supplementation prescription in the community: a qualitative study. Clin Nutr ESPEN. 2020;36:116-127. doi:10.1016/j.clnesp.2020.01.006

35. Beckingsale L, Fairbairn K, Morris C. Integrating dietitians into primary health care: benefits for patients, dietitians and the general practice team. J Prim Health Care. 2016;8:372-380. doi:10.1071/ HC16018

36. European Ageing Network. Promoting well-nutrition in elderly care. Available from: file://C:/Users/LindnerSo/Downloads/2021-06Promoting-well-nutrition-in-elderly-care-FINAL\%20(1).pdf. Accessed November 19, 2021.

37. Valentijn PP, Schepman SM, Opheij W, Bruijnzeels MA. Understanding integrated care: a comprehensive conceptual framework based on the integrative functions of primary care. Int J Integr Care. 2013;13:e010. doi:10.5334/ijic.886 
38. Kennelly S, Kennedy NP, Corish CA, Flanagan-Rughoobur G, Glennon-Slattery C, Sugrue S. Sustained benefits of a community dietetics intervention designed to improve oral nutritional supplement prescribing practices. J Hum Nutr Diet. 2011;24(5):496-504. doi:10.1111/j.1365-277X.2011.01197.x

39. Munk T, Svendsen JA, Knudsen AW, Østergaard TB, Beck AM. Effect of nutritional interventions on discharged older patients: study protocol for a randomized controlled trial. Trials. 2020;21 (1):365. doi:10.1186/s13063-020-04301-6

40. Maeda K, Koga T, Akagi J. Nutritional variables predict chances of returning home and activities of daily living in post-acute geriatric care. Clin Interv Aging. 2018;13:151-157. doi:10.2147/CIA.S154129

41. Vearing R, Casey S, Zaremba C, et al. Evaluation of the impact of a post-hospital discharge Transitional Aged Care Service on frailty, malnutrition and functional ability. Nutr Diet. 2019;76(4):472-479. doi:10.1111/1747-0080.12511
42. Fried LP, Tangen CM, Walston J, et al. Frailty in older adults: evidence for a phenotype. J Gerontol Mar. 2001;56(3):M146-56. doi:10.1093/gerona/56.3.M146

43. Roberts HC, Lim SER, Cox NJ, Ibrahim K. the challenge of managing undernutrition in older people with frailty. Nutrients. 2019;11 (4):808. doi:10.3390/nu11040808

44. Murphy J, Mayor A, Forde E. Identifying and treating older patients with malnutrition in primary care: the MUST screening tool. $\mathrm{Br}$ J Gen Pract. 2018;68(672):344-345. doi:10.3399/bjgp18X697853

45. Pedersen JL, Damsgaard EM. Undernutrition in older people. A narrative review. Available from: https:/www.smr-conference.dk/ SysSiteAssets/abstracts-topics/undernutrition.pdf. Accessed September 30, 2021

46. Swift JA, Tischler V. Qualitative research in nutrition and dietetics: getting started. J Human Nutr Diet. 2010;23:559-566. doi:10.1111/ j.1365-277X.2010.01116.x
Clinical Interventions in Aging

\section{Publish your work in this journal}

Clinical Interventions in Aging is an international, peer-reviewed journal focusing on evidence-based reports on the value or lack thereof of treatments intended to prevent or delay the onset of maladaptive correlates of aging in human beings. This journal is indexed on PubMed Central, MedLine, CAS, Scopus and the Elsevier
Dovepress

Bibliographic databases. The manuscript management system is completely online and includes a very quick and fair peer-review system, which is all easy to use. Visit http://www.dovepress.com/ testimonials.php to read real quotes from published authors. 